Pacific Journal of Mathematics

ON THE METRIC THEORY OF DIOPHANTINE 


\title{
ON THE METRIC THEORY OF DIOPHANTINE APPROXIMATION
}

\author{
JEFFREY D. VAALER
}

A conjecture of Duffin and Schaeffer states that

$$
\sum_{n=2}^{\infty} \alpha_{n} \varphi(n) n^{-1}=+\infty
$$

is a necessary and sufficient condition that for almost all real $x$ there are infinitely many positive integers $n$ which satisfy $|x-a / n|<\alpha_{n} n^{-1}$ with $(a, n)=1$. The necessity of the condition is well known. We prove that the condition is also sufficient if $\alpha_{n}=O\left(n^{-1}\right)$.

1. Introduction. Let $\left\{\alpha_{n}\right\}, n=2,3,4, \cdots$, be a sequence of real numbers satisfying $0 \leqq \alpha_{n} \leqq 1 / 2$. We consider the problem of determining a sufficient condition on the sequence $\left\{\alpha_{n}\right\}$ so that for almost all real $x$ the inequality

$$
\left|x-\frac{a}{n}\right|<\frac{\alpha_{n}}{n}
$$

holds for infinitely many pairs of relatively prime integers $a$ and $n$. We note that there is no loss of generality if we restrict $x$ to the interval $I=[0,1]$. Let $\lambda$ be Lebesgue measure on $I$ and define

$$
E_{n}=\bigcup_{\substack{a=1 \\(a, n)=1}}^{n}\left(\frac{a-\alpha_{n}}{n}, \frac{a+\alpha_{n}}{n}\right),
$$

where $(a, n)$ denotes the greatest common divisor of $a$ and $n$. Then our problem is to determine a sufficient condition on $\left\{\alpha_{n}\right\}$ so that

$$
\lim _{N \rightarrow \infty} \lambda\left\{\bigcup_{n=N}^{\infty} E_{n}\right\}=1 .
$$

It is clear that $\lambda\left(E_{n}\right)=2 \alpha_{n} \varphi(n) / n$ where $\varphi$ is Euler's function. Thus by the Borel-Cantelli lemma,

$$
\sum_{n=2}^{\infty} \lambda\left(E_{n}\right)=2 \sum_{n=2}^{\infty} \frac{\alpha_{n} \varphi(n)}{n}=+\infty
$$

is a necessary condition for (1.2) It has been conjectured by Duffin and Schaeffer [4] that (1.3) is also a sufficient condition for (1.2), but this has never been proved. Khintchine [7] showed that if $n \alpha_{n}$ is a decreasing function of $n$ then (1.3) implies (1.2). (Actually, Khintchine's result is usually stated in a different but equivalent 
form.) Duffin and Schaeffer [4] improved Khintchine's theorem by showing that if

$$
\sum_{n=2}^{N} \frac{\alpha_{n} \varphi(n)}{n} \geqq c \sum_{n=2}^{N} \alpha_{n}
$$

for some constant $c>0$ and for arbitrarily large values of $N$ then (1.3) implies (1.2). More recently Erdös [5] proved the following special case of the Duffin-Schaeffer conjecture:

ERDös' THEOREM. If $\alpha_{n}=0$ or $\varepsilon / n$ for all $n$ and some $\varepsilon>0$, then (1.3) implies (1.2).

In the present paper we generalize Erdös' theorem by proving

$$
\text { Theorem 1. If } \alpha_{n}=O\left(n^{-1}\right) \text { then (1.3) implies (1.2). }
$$

If the sets $E_{n}$ were pairwise independent, that is if $\lambda\left(E_{n} \cap E_{m}\right)=$ $\lambda\left(E_{n}\right) \lambda\left(E_{m}\right)$ for all $n \neq m$, then (1.3) would imply (1.2) by the "divergence part" of the Borel-Cantelli lemma, (Chung [3], Theorem 4.3.2). In general the sets $E_{n}$ are not pairwise independent. However, by using some weaker bound on $\lambda\left(E_{n} \cap E_{m}\right)$ we can still deduce the desired result. This is also the approach used in [4] and [5]. We give a simpler treatment of this part of the problem by employing a theorem of Gallagher. Let $Z$ denote a finite subset of $\{2,3,4, \cdots\}$ and define $\Lambda(Z)$ by

$$
\Lambda(Z)=\sum_{n \in Z} \lambda\left(E_{n}\right)
$$

Then we obtain Theorem 1 from

THEOREM 2. Suppose there exists an integer $K \geqq 2$ and a real number $\eta>0$ such that the following condition holds: every finite subset $Z$ of $\{K, K+1, K+2, \cdots\}$ with $0 \leqq \Lambda(Z) \leqq \eta$ also satisfies

$$
\sum_{\substack{n \in Z \\ n \neq m \\ n \neq Z}} \sum_{\substack{n \\ n}} \lambda\left(E_{n} \cap E_{m}\right) \leqq \Lambda(Z) \text {. }
$$

Then (1.3) implies (1.2).

Proof. We assume that (1.3) holds. By a result of Gallagher [6], the value of $\lim _{N \rightarrow \infty} \lambda\left\{\bigcup_{n=N}^{\infty} E_{n}\right\}$ is either zero or one. We suppose that

$$
\lim _{N \rightarrow \infty} \lambda\left\{\bigcup_{n=N}^{\infty} E_{n}\right\}=0
$$


If $\lim \sup _{n \rightarrow \infty} \lambda\left(E_{n}\right)=\xi>0$ then $\lambda\left\{\bigcup_{n=N}^{\infty} E_{n}\right\} \geqq \xi$ for all $N$, which contradicts (1.6). Thus we may assume that

$$
\lim _{n \rightarrow \infty} \lambda\left(E_{n}\right)=0 \text {. }
$$

Now choose $M$ so large that

$$
\lambda\left\{\bigcup_{n=M}^{\infty} E_{n}\right\} \leqq \frac{1}{4} \eta .
$$

Let $J=\max \{K, M\}$. From (1.3) and (1.7) it follows that there exists a finite subset $Z$ of $\{J, J+1, J+2, \cdots\}$ such that

$$
\frac{2}{3} \eta \leqq \Lambda(Z) \leqq \eta
$$

But then by a simple sieve argument

$$
\begin{aligned}
\frac{1}{4} \eta & \geqq \lambda\left\{\bigcup_{n \in Z} E_{n}\right\} \\
& \geqq \sum_{n \in Z} \lambda\left(E_{n}\right)-\frac{1}{2} \sum_{\substack{n \in Z \\
n \neq m}} \sum_{\substack{n \in Z \\
n}} \lambda\left(E_{n} \cap E_{m}\right) \\
& \geqq \Lambda(Z)-\frac{1}{2} \Lambda(Z) \\
& \geqq \frac{1}{3} \eta,
\end{aligned}
$$

which is impossible.

The remainder of our paper will consist of showing that if $\alpha_{n}=O\left(n^{-1}\right)$ then the hypotheses in Theorem 2 are satisfied. In fact we will prove the following result, which gives a stronger estimate than we require.

THEOREM 3. If $\alpha_{n} \leqq C n^{-1}$ for all $n$ and some $C>0$ then there exists a real number $\eta_{0}>0$ such that the following condition holds: if $Z$ is a finite subset of $\{2,3,4, \cdots\}$ with $0<\Lambda(Z) \leqq \eta_{0}$, then

$$
\begin{aligned}
& \sum_{\substack{n \in Z \\
n \neq m}} \sum_{\substack{m \in Z \\
n \neq m}} \lambda\left(E_{n} \cap E_{m}\right) \\
& \ll \Lambda(Z)^{2}\left(\log \log \left\{\Lambda(Z)^{-1}\right\}\right)^{2} .
\end{aligned}
$$

Here, and elsewhere in this paper, the constant implied by $\ll$ is absolute.

Our proof of Theorem 3 is modeled after Erdös' proof in [5]. In $\S 2$ we give several lemmas for later use. We then split the sum 
on the left of (1.8) into three parts which are estimated in $\S \S 3$ and 4. It is in $\S 4$ that the main difficulty occurs. Indeed it is only there that we require the hypothesis $\alpha_{n} \leqq C n^{-1}$.

We remark that Catlin $[1,2]$ has recently found a connection between (1.1) and the problem of approximating almost all $x$ by fractions $a / n$ which are not necessarily reduced. Thus our results also have implications for this problem. We note, however, that the proof of Theorem 3 in [1] contains a serious error.

2. Preliminary lemmas. Throughout the remainder of this paper $p$ will denote a prime. Thus $\sum_{p\rfloor_{n}}$ is a sum over prime divisors of $n$ and $\pi(x)=\sum_{p \leqq x} 1$ is the number of primes not exceeding $x$. For each integer $n \geqq 2$ we define $g(n)$ to be the smallest positive integer $v$ such that

$$
\sum_{\substack{p \mid n \\ p>v}} \frac{1}{p}<1
$$

If $g(n)=v$ then

$$
\begin{aligned}
\prod_{\substack{p \mid n \\
p \leqq v}}\left(1-\frac{1}{p}\right) & =\frac{\varphi(n)}{n} \prod_{\substack{p \mid n \\
p>v}}\left(1-\frac{1}{p}\right)^{-1} \\
& \leqq \frac{\varphi(n)}{n} \exp \left\{\sum_{\substack{p \nmid n \\
p>v}} \frac{1}{p}+\sum_{p} \sum_{j=2}^{\infty} j^{-1} p^{-j}\right\} \\
& \ll \frac{\varphi(n)}{n} .
\end{aligned}
$$

It follows from the theorem of Mertens that

$$
1 \ll \frac{\varphi(n)}{n} \log (1+v) .
$$

Next let $\xi>0, x>0$ and let $v$ be a positive integer. We define $N(\xi, v, x)$ to be the number of integers $n \leqq x$ which satisfy

$$
\sum_{\substack{p \nmid n \\ p \geqq v}} \frac{1}{p} \geqq \xi
$$

We then have the following estimate of Erdös [5].

Lemma 4. For any $\varepsilon>0$ and $\xi>0$ there exists a positive integer $v_{0}=v_{0}(\xi, \varepsilon)$ such that for all $x>0$ and all $v \geqq v_{0}$,

$$
N(\xi, v, x) \leqq x \exp \left\{-v^{\beta(1-\varepsilon)}\right\}
$$

where $\log \beta=\xi$. 
Proof. We may assume that $0<\varepsilon<\left(1-e^{-\xi}\right)$. Let

$$
p_{1}<p_{2}<\cdots<p_{M}
$$

be the set of all primes in $[v, w]$, where $w=v^{\beta(1-\varepsilon / 3)}$. If $v$ is sufficiently large then $M \geqq \pi(w)-\pi(v) \geqq v^{\beta(1-2 \varepsilon / 3)}$.

We split the integers $n \leqq x$ which satisfy (2.3) into two classes. In the first class are integers $n$ with $M$ prime factors in the interval $[v, \exp (w)]$. The number of such integers is clearly less than

$$
x\left(\sum_{v \leqq p \leqq \exp (w)} \frac{1}{p}\right)^{M} / M ! \leqq x\left(c_{1} \log w\right)^{M} / M !
$$

for some constant $c_{1}>0$. Using Stirling's formula this is easily seen to be

$$
\ll x \exp (-M) \ll x \exp \left\{-v^{\beta(1-2 \varepsilon / 3)}\right\}
$$

for sufficiently large $v$.

Next we observe that

$$
\begin{aligned}
\sum_{j=1}^{M} \frac{1}{p_{j}} & =\sum_{v \leqq p \leqq w} \frac{1}{p}=\log \left(\frac{\log w}{\log v}\right)+o(1) \\
& =\xi+\log (1-\varepsilon / 3)+o(1) \\
& \leqq \xi-\varepsilon / 3
\end{aligned}
$$

for sufficiently large $v$. The integers $n \leqq x$ which satisfy (2.3) and which have fewer than $M$ prime factors in $[v, \exp (w)]$ must therefore satisfy

$$
\frac{3}{\varepsilon} \sum_{\substack{p \nmid n \\ p>\exp w}} \frac{1}{p} \geqq 1
$$

The number of such integers $n$ is

$$
\begin{aligned}
& \leqq \frac{3}{\varepsilon} \sum_{n \leqq x} \sum_{\substack{p \nmid n \\
p>\exp w}} \frac{1}{p}=\frac{3}{\varepsilon} \sum_{p>\exp w} \frac{1}{p}\left[\frac{x}{p}\right] \\
& \ll \frac{x}{\varepsilon} \sum_{p>\exp w} \frac{1}{p^{2}} \ll \frac{x}{\varepsilon} \exp (-w) .
\end{aligned}
$$

The bound (2.4) now follows from (2.5) and (2.7).

We now suppose that $g(n)=u \leqq v$. For each $\xi>0$ we split the divisors $d$ of $n$ into two classes, $A_{n}(\xi, v)$ and $B_{n}(\xi, v)$. We say that $d$ is in $A_{n}(\xi, v)$ if

$$
\sum_{\substack{p \mid d \\ p \geq v}} \frac{1}{p} \geqq \xi
$$


The class $B_{n}(\xi, v)$ consists of divisors which do not satisfy (2.8).

Lemma 5. For any $\varepsilon>0$ and any $\xi>0$ there exists a positive integer $v_{0}=v_{0}(\xi, \varepsilon)$ such that if $g(n)=u \leqq v$ and $v \geqq v_{0}$ then

$$
\sum_{d \in A_{n^{\prime}(\xi, v)}} \frac{1}{d} \leqq(\log (1+u)) \exp \left\{-v^{\beta(1-s)}\right\}
$$

where $\log \beta=\xi$.

Proof. Let $v, w$ and $M$ be as in the proof of Lemma 4. For any collection $\mathscr{S P}^{\circ}$ of $M$ primes in $[v, \infty)$ we have

$$
\sum_{p \in \mathscr{S}} \frac{1}{p} \leqq \sum_{j=1}^{M} \frac{1}{p_{j}} \leqq \xi-\varepsilon / 3
$$

for sufficiently large $v$, as in (2.6). Thus if $d \in A_{n}(\xi, v)$ then $d$ must have at least $M$ prime factors in $[v, \infty)$. Let $q_{1}, q_{2}, \cdots, q_{J}$ be the prime factors of $n$ which are greater than or equal to $v$. If $J \leqq M$ then $A_{n}(\xi, v)$ is empty. Otherwise

$$
\sum_{d \in A_{n}(\hat{\xi}, v)} \frac{1}{d} \leqq\left(\sum_{d \mid n} \frac{1}{d}\right)\left(\sum_{j=1}^{J} \frac{1}{q_{j}}\right)^{M} / M ! .
$$

Since $g(n)=u \leqq v$ we have

$$
\left(\sum_{\jmath=1}^{I} \frac{1}{q_{j}}\right)^{M} / M ! \leqq(M !)^{-1} \ll \exp \left\{-v^{\beta(1-2 \varepsilon / 3)}\right\} .
$$

Also,

$$
\sum_{d \backslash n} \frac{1}{d} \leqq \prod_{p \leqq n}\left(1-\frac{1}{p}\right)^{-1} \prod_{\substack{p \nmid n \\ p>u}}\left(1-\frac{1}{p}\right)^{-1} \ll \log (1+u)
$$

by the theorem of Mertens.

Let $\sum_{m}(v)$ denote a sum over integers $m$ which satisfy $g(m)=v$.

LEMMA 6. Let $\varepsilon>0$. Then there exists a constant $v_{0}=v_{0}(\varepsilon)$ such that the following inequalities hold: if $x>0$ and $y \geqq 2$, if $g(n)=u \leqq v$ and $v \geqq v_{0}$, then

$$
\sum_{\substack{m(v) \\(n, m) x<m<(n, m) x y}} m^{-1} \leqq(\log 1+u)(\log y) \exp \left\{-v^{\beta(1-\varepsilon)}\right\}
$$

and

$$
\sum_{\substack{m(v) \\(n, m)^{-1} x<m<\langle n, m)^{-1} x y}} m^{-1} \leqq(\log 1+u)(\log y) \exp \left\{-v^{\beta(1-\varepsilon)}\right\},
$$


where $\beta=e^{1 / 2}$.

Proof. The proofs of the two inequalities are virtually identical, so we prove only (2.10). We have

$$
\sum_{\substack{m(v) \\(n, m) x<m<(n, m) x y}} m^{-1}=\sum_{\substack{d \mid n \\(n, m)=d \\ d x<m<d x y}} \sum_{\substack{m(v) \\ d x<m}} m^{-1} .
$$

If $(n, m)=d$ we write $m=d m^{\prime}$. Then by Lemma 5 with $\xi=1 / 2$,

$$
\begin{aligned}
\sum_{d \in A_{n}(1 / 2, v)} & \sum_{\substack{m(v) \\
(n, m)=d \\
d x<m<d x y}} m^{-1} \\
\leqq & \sum_{d \in A_{n}(1 / 2, v)} d^{-1} \sum_{\substack{m^{\prime} \\
x<m^{\prime}<x y}}\left(m^{\prime}\right)^{-1} \\
\leqq & (\log 1+u)(\log y) \exp \left\{-v^{\beta(1-\varepsilon / 2)}\right\},
\end{aligned}
$$

for sufficiently large $v$.

If $d \in B_{n}(1 / 2, v)$ then

$$
\begin{aligned}
1 & \leqq \sum_{\substack{p \mid m \\
p \geqq v}} p^{-1} \leqq \sum_{\substack{p \mid d \\
p \geqq v}} p^{-1}+\sum_{\substack{p \mid m \\
p \geqq v}} p^{-1} \\
& <\frac{1}{2}+\sum_{\substack{p \mid m, m \\
p \geqq v}} p^{-1}
\end{aligned}
$$

and so

$$
\sum_{\substack{p|m, p| \geq v}} p^{-1}>\frac{1}{2}
$$

By Lemma 4

$$
\sum_{\substack{m^{\prime} \\ n<m^{\prime} \leqq 2 x}}\left(m^{\prime}\right)^{-1} \leqq x^{-1} N\left(\frac{1}{2}, v, 2 x\right) \leqq 2 \exp \left\{-v^{\beta(1-\varepsilon / 2)}\right\}
$$

for sufficiently large $v$, where the sum on the left of (2.13) is over $m^{\prime}$ satisfying (2.12). Hence

$$
\begin{aligned}
& \sum_{d \in B(1 / 2, v)} \sum_{\substack{m^{\prime}(v) \\
(n, m) d \\
d x<m<d x y}} m^{-1} \\
& \leqq \\
& \sum_{d \in B} \sum_{n^{(1 / 2, v)}} d^{-1} \sum_{\substack{m^{\prime} \\
x<m^{\prime}<x y}}\left(m^{\prime}\right)^{-1} \\
& \leqq \sum_{d \in B_{n}(1 / 2, v)} d^{-1}(\log y) \exp \left\{-v^{\beta(1-\varepsilon / 2)}\right\} \\
& \ll \log (1+u)(\log y) \exp \left\{-v^{\beta(1-\varepsilon / 2)}\right\}
\end{aligned}
$$

for sufficiently large $v$.

3. First estimates. In this section we begin our proof of Theorem 
3. For $n \neq m$ we define

$$
\begin{aligned}
& \delta=\delta(n, m)=2 \min \left\{\frac{\alpha_{n}}{n}, \frac{\alpha_{m}}{m}\right\}, \\
& \Delta=\Delta(n, m)=2 \max \left\{\frac{\alpha_{n}}{n}, \frac{\alpha_{m}}{m}\right\},
\end{aligned}
$$

and

$$
t=t(n, m)=\max \{g(n), g(m)\} .
$$

We write $\sum_{a=1}^{n_{*}}$ and $\sum_{b=1}^{m_{*}}$ for sums over integers prime to $n$ and $m$ respectively. Thus

$$
\begin{aligned}
& \lambda\left(E_{n} \cap E_{m}\right) \\
& =\sum_{a=1}^{n_{*}} \sum_{b=1}^{m_{*}} \lambda\left\{\left(\frac{a-\alpha_{n}}{n}, \frac{a+\alpha_{n}}{n}\right) \cap\left(\frac{b-\alpha_{m}}{m}, \frac{b+\alpha_{m}}{m}\right)\right\} \\
& \leqq \delta(n, m) \sum_{\substack{a=1 \\
|a| n-b|m|<\Delta(n, m)}}^{n^{*}} \sum_{\substack{b=1 \\
m_{*}}} 1 \\
& =\delta \sum_{\substack{a=1 \\
|a m-b n|<n m \Delta}}^{n_{*}} \sum_{\substack{b=1 \\
m_{*}}}^{1} 1 \text {. }
\end{aligned}
$$

For each integer $u$ we define $H(u)$ to be the number of pairs $\{a, b\}$ which satisfy

$$
\begin{aligned}
& a m-b n=u, \quad 1 \leqq a \leqq n, \quad(a, n)=1, \\
& 1 \leqq b \leqq m, \quad(b, m)=1 .
\end{aligned}
$$

From (3.1) it follows that

$$
\lambda\left(E_{n} \cap E_{m}\right) \leqq \delta \sum_{\substack{u \\|u|<n m \Delta}} H(u) .
$$

Let $d=(n, m)$. It is clear that $H(0)=0$ and if $d \nmid u$ then $H(u)=0$. Thus in estimating the right hand side of (3.2) we may assume that

$$
d<n m \Delta
$$

and restrict ourselves to integers $u$ which are divisible by $d$. We write $|u|=d d_{u} u_{1}$, where the prime divisors of $d_{u}$ also divide $d$ and $\left(d, u_{1}\right)=1$. Obviously this decomposition is unique. It is shown in [5] that if either $\left(u_{1}, n m d^{-1}\right)>1$ or $\left(d_{u}, n m d^{-2}\right)>1$ then $H(u)=0$. Hence we may further restrict ourselves to integers $u$ which satisfy

$$
\left(u_{1}, n m d^{-1}\right)=\left(d_{u}, n m d^{-2}\right)=1 .
$$

For such $u$ we have the estimate 


$$
\begin{aligned}
H(u) & \leqq d \prod_{\substack{p \mid d \\
p \nmid d u^{n m m d^{-2}}}}\left(1-\frac{2}{p}\right) \prod_{\substack{p|d \\
p| d u^{n m}-d^{-2}}}\left(1-\frac{1}{p}\right) \\
& \leqq \varphi(d) \prod_{\substack{p \mid d \\
p \nmid n m d^{-2}}}\left(1-\frac{1}{p}\right) \prod_{\substack{p \mid d_{u} \\
p}}\left(1-\frac{1}{p}\right)^{-1}
\end{aligned}
$$

from [5].

Next let $\mathscr{P}_{0}$ be the set of primes $p$ which divide $d$ but do not divide $n m d^{-2}$. We split $\mathscr{P}_{0}$ into disjoint subsets $\mathscr{P}_{1}$ and $\mathscr{P}_{2}$ consisting of primes satisfying $p \leqq t$ and $p>t$ respectively. Let $\mathscr{S}_{j}$ be the set of positive integers whose prime divisors are in $\mathscr{P}_{j}$, for $j=0,1,2$. From (3.4) we may assume that $d_{u} \in \mathscr{S}_{0}$ and hence that $d_{u}$ is uniquely represented as $d_{u}=s_{1} s_{2}$ with $s_{1} \in \mathscr{S}_{1}$ and $s_{2} \in \mathscr{S}_{2}$. Thus

$$
\begin{aligned}
H(u) & \leqq \varphi(d) \prod_{p \in \mathscr{S}_{0}}\left(1-\frac{1}{p}\right) \prod_{p \mid s_{1} s_{2}}\left(1-\frac{1}{p}\right)^{-1} \\
& \leqq \varphi(d) \prod_{p \in \mathscr{V}_{1}}\left(1-\frac{1}{p}\right) \prod_{p \mid s_{1}}\left(1-\frac{1}{p}\right)^{-1} .
\end{aligned}
$$

Now $|u|=d d_{u} u_{1}=d s_{1} s_{2} u_{1}$ where the set of primes which divide $s_{1}, s_{2}$, and $u_{1}$ are all distinct. Therefore if we set $k=s_{2} u_{1}$ then $k$ is relatively prime to

$$
Q=\prod_{\substack{p \mid m m d^{-1} \\ p \leqq t}} p
$$

by (3.4) and the definition of $\mathscr{P}_{2}$. From (3.2) and (3.6) we obtain

$$
\begin{aligned}
\lambda\left(E_{n} \cap E_{m}\right) & \leqq \delta \sum_{\substack{u \\
|u|<n m \Delta}} H(u) \\
& =\delta \sum_{s_{1} \in \mathscr{S}_{1}} \sum_{1 \leqq k \leqq\left(n m \Delta \mid d s_{1}\right)}^{*}\left\{H\left(-d s_{1} k\right)+H\left(d s_{1} k\right)\right\} \\
& \leqq 2 \delta \varphi(d) \prod_{p \in \mathscr{S}_{1}}\left(1-\frac{1}{p}\right)\left\{\sum_{s_{1} \in \mathscr{S}_{1}} \prod_{p \mid s_{1}}\left(1-\frac{1}{p}\right)^{-1} \sum_{1 \leqq k \leqq\left\langle n m \Delta / d s_{1}\right)}^{*} 1\right\},
\end{aligned}
$$

where $(k, Q)=1$ in the sum $\Sigma^{*}$.

By the prime number theorem there exists an absolute constant $b$ such that

$$
\pi(y) \log 2 y+\log \log y \leqq y \log 3
$$

for all $y \geqq b$. Throughout the remainder of this section we shall assume that

$$
t=t(n, m) \geqq b \quad \text { and } \quad n m \Delta \geqq 3^{t} d .
$$

Then by the sieve of Erathosthenes 


$$
\sum_{1 \leqq k \leqq\left(n m \Delta / d s_{1}\right)}^{*} 1 \leqq \frac{n m \Delta}{d s_{1}} \prod_{p \mid Q}\left(1-\frac{1}{p}\right)+2^{\pi(t)} .
$$

If $s_{1} \leqq t^{\pi(t)}$ then using (3.8) and (3.9) we have

$$
\begin{aligned}
2^{\pi(t)} & \leqq 3^{t} t^{-\pi(t)} \log ^{-1} t \\
& \ll \frac{n m \Delta}{d s_{1}} \prod_{p \mid Q}\left(1-\frac{1}{p}\right) .
\end{aligned}
$$

It follows that if we sum over $s_{1} \leqq t^{\pi(t)}$ on the right hand side of (3.7) we obtain the upper bound

$$
\begin{aligned}
2 \delta \varphi(d) & \prod_{p \in \mathscr{P}_{1}}\left(1-\frac{1}{p}\right) \sum_{s_{1} \leq t \pi(t)} s_{1} \varphi\left(s_{1}\right)^{-1} \sum_{1 \leqq k \leqq\left(n m \Delta / d s_{1}\right)}^{*} 1 \\
& \ll \delta \frac{\varphi(d)}{d} n m \Delta \prod_{p \mid Q}\left(1-\frac{1}{p}\right) \prod_{p \in \mathscr{P}_{1}}\left(1-\frac{1}{p}\right) \sum_{s_{1} \in \mathscr{S}_{1}} \varphi\left(s_{1}\right)^{-1} \\
& \ll \alpha_{n} \alpha_{m} \frac{\varphi(d)}{d} \prod_{p \mid n m d^{-1}}\left(1-\frac{1}{p}\right) \prod_{p \in \mathscr{T}_{1}}\left(1+\frac{1}{p(p-1)}\right) \\
& \ll \frac{\alpha_{n} \varphi(n)}{n} \frac{\alpha_{m} \varphi(m)}{m} \ll \lambda\left(E_{n}\right) \lambda\left(E_{m}\right) .
\end{aligned}
$$

Now if $s_{1}>t^{\pi(t)}$ we easily see that for some prime $p \in \mathscr{P}_{1}$ and some integer $\gamma \geqq 2$ we must have $p^{\gamma} \mid s_{1}, p^{\gamma}>t p \leqq t$. By considering the cases where $\gamma$ is even or odd it follows that $s_{1}$ is divisible by a square greater than $t^{2 / 3}$. Thus summing over $s_{1}>t^{\pi(t)}$ in (3.7) we obtain

$$
\begin{aligned}
2 \delta \varphi(d) \prod_{p \in \mathscr{O}_{1}} & \left(1-\frac{1}{p}\right) \sum_{t^{\pi}(t)<s_{1}} s_{1} \varphi\left(s_{1}\right)^{-1} \sum_{1 \leqq k \leqq\left(n m \Delta / d s_{1}\right)}^{*} 1 \\
& \leqq 2 \delta \varphi(d) \sum_{t^{\pi}(t)<s_{1} 1 \leqq k \leqq\left(n m \Delta / d s_{1}\right)} 1 \\
& \leqq 2 \delta \varphi(d) \sum_{r=\left[t^{1 / 3}\right]} \sum_{1 \leqq j \leqq(n m \Delta / d)} 1 \\
& \leqq 2 \delta \varphi(d) \frac{n m \Delta}{d} \sum_{r=\left[t^{1 / 3}\right]}^{\infty} r^{-2 \mid j} \\
& \ll \alpha_{n} \alpha_{m} t^{-1 / 3} \ll\left(\alpha_{n} \frac{\varphi(n)}{n}\right)\left(\alpha_{m} \frac{\varphi(m)}{m}\right) t^{-1 / 3} \log ^{2} t \\
& \ll \lambda\left(E_{n}\right) \lambda\left(E_{m}\right) .
\end{aligned}
$$

Putting the estimates in (3.10) and (3.11) together, it follows that

$$
\lambda\left(E_{n} \cap E_{m}\right) \ll \lambda\left(E_{n}\right) \lambda\left(E_{m}\right)
$$

for all pairs $\{n, m\}, n \neq m$, which satisfy (3.9). 
4. Second estimates. Let $Z$ be a finite subset of $\{2,3,4, \cdots\}$ with $\Lambda(Z)$ defined by (1.5). We choose $\varepsilon$ in Lemma 6 so that $e^{1 / 2}(1-\varepsilon)=3 / 2$. This determines an absolute constant $v_{0}$ such that (2.10) and (2.11) hold for all $v \geqq v_{0}$. We then define $\eta_{0}$ by

$$
\eta_{0}=\exp \left\{-\max \left(b, C, v_{0}\right)\right\}
$$

and assume that $0<\Lambda(Z) \leqq \eta_{0}$.

Next we write

$$
\sum_{\substack{n \in Z \\ n \neq m}} \sum_{m \in Z} \lambda\left(E_{n} \cap E_{m}\right)=S_{1}+S_{2}
$$

where $S_{1}$ is the sum over pairs $\{n, m\}$ which satisfy (3.9) and $S_{2}$ is the sum over the remaining pairs $\{n, m\}$ which do not satisfy (3.9). We apply (3.12) to obtain the estimate

$$
S_{1} \ll \sum_{n \in Z} \sum_{m \in Z} \lambda\left(E_{n}\right) \lambda\left(E_{m}\right)=\Lambda(Z)^{2} .
$$

Thus it remains only to bound $S_{2}$.

From (2.2) and (3.7) we have

$$
\begin{aligned}
\lambda\left(E_{n} \cap E_{m}\right) & \\
& \leqq 2 \delta \varphi(d) \frac{n m \Delta}{d} \prod_{p_{1} \in \mathscr{O}_{1}}\left(1-\frac{1}{p_{1}}\right) \sum_{s_{1} \in \mathscr{S}_{1}} \varphi\left(s_{1}\right)^{-1} \\
& \ll \alpha_{n} \alpha_{m} \ll \log ^{2}(1+t) \lambda\left(E_{n}\right) \lambda\left(E_{m}\right) .
\end{aligned}
$$

Hence if we set $L=-\log \{\Lambda(Z)\}$ and sum over pairs $\{n, m\}$ which satisfy $t<L$ we obtain

$$
\begin{aligned}
\sum_{\substack{n \in Z \\
n \neq m \\
t<L}} \sum_{\substack{m \\
t<L}} \lambda\left(E_{n} \cap E_{m}\right) \\
\quad \ll \Lambda(Z)^{2}\left(\log \log \left\{\Lambda(Z)^{-1}\right\}\right)^{2} .
\end{aligned}
$$

Now for any pair $\{n, m\}$ in the sum $S_{2}$ we have either $t<b$ or $n m \Delta<$ $3^{t} d$, where $d=(n, m)$. But from (4.1) we have $b \leqq L$ so that terms for which $t<b$ are already included in (4.4). Therefore the only sum which we need to bound is

$$
S_{3}=\sum_{\substack{n \in Z \\ n \neq m \\ n \neq m}} \sum_{\substack{m \\ \text { nat }}} \lambda\left(E_{n} \cap E_{m}\right)
$$

where each pair $\{n, m\}$ satisfies $t \geqq L$ and (using (3.3))

$$
d<n m \Delta<3^{t} d \text {. }
$$

We have 


$$
\begin{aligned}
S_{3} & \ll \sum_{v=L}^{\infty} \sum_{u=1}^{v}\left\{\sum_{\substack{n^{(u)} \\
(n, m)<n m \Delta<3^{v}(v)}} \alpha_{n} \alpha_{m}\right\} \\
& \ll \sum_{v=L}^{\infty} \log (1+v) \sum_{u=1}^{v}\left\{\sum_{n^{(}(u)} \lambda\left(E_{n}\right) \quad \sum_{\substack{m^{(v)} \\
(n, m)<n m<3^{v}(n, m)}} \alpha_{m}\right\},
\end{aligned}
$$

where we have used (2.2) and (4.3). Our objective it to establish

$$
\sum_{\substack{m(v) \\(n, m)<n m \Delta<3^{v}(n, m)}} \alpha_{m} \ll C v(\log 1+v) \exp \left\{-v^{3 / 2}\right\}
$$

for the sums on the right of (4.6), that is for fixed $n, g(n)=u \leqq v$ and $v \geqq v_{0}$. To accomplish this we consider two cases.

If $\alpha_{m} / m \leqq \alpha_{n} / n$ then the condition $(n, m)<n m \Delta<3^{v}(n, m)$ becomes $(n, m)<2 m \alpha_{n}<3^{v}(n, m)$. Clearly we may assume that $\alpha_{n}>0$ so that by (2.10) we have

$$
\begin{aligned}
\sum_{\substack{m(v) \\
(n, m)<2 m \alpha_{n}<3^{v}(n, m)}} \alpha_{m} & \leqq C \sum_{\substack{m(v) \\
(n, m)<2 m \alpha_{n}<3\left(n, m^{v}\right)}} m^{-1} \\
& \ll C v(\log 1+v) \exp \left\{-v^{3 / 2}\right\} .
\end{aligned}
$$

If $\alpha_{n} / n<\alpha_{m} / m$ then the condition becomes

$$
(n, m)<2 n \alpha_{m}<3^{v}(n, m) \text {. }
$$

Since $\alpha_{k} \leqq C k^{-1}$ we may partition $Z$ into disjoint classes $W_{j}, j=$ $0,1,2, \cdots$, defined by

$$
W_{j}=\left\{k \in Z: C 2^{-j-1}<k \alpha_{k} \leqq C 2^{-j}\right\} .
$$

If $m \in W_{j}$ and $m$ satisfies (4.8) then we have

$$
2^{-1}(n, m)<n m^{-1} C 2^{-j}<3^{v}(n, m)
$$

and so

$$
C 2^{-j} n 3^{-v}(n, m)^{-1}<m<C 2^{1-j} n(n, m)^{-1} \text {. }
$$

Therefore we may apply (2.11) with $x=C 2^{-j} n 3^{-v}$ and $y=2\left(3^{v}\right)$ to obtain

$$
\begin{aligned}
\sum_{\substack{m(v) \\
(n, m)<2 n \alpha_{m}<3^{v}(n, m)}} \alpha_{m} & \leqq C \sum_{j=0}^{\infty} 2^{-j} \sum_{\substack{m(v) \\
m \in W_{j}}}^{*} m^{-1} \\
& \ll C v(\log 1+v) \exp \left\{-v^{3 / 2}\right\},
\end{aligned}
$$

where $\Sigma^{*}$ indicates a sum over $m$ 's which satisfy (4.9). This proves (4.7).

By using (4.1), (4.6), and (4.7) we find that 


$$
\begin{aligned}
S_{3} & \ll C \sum_{v=L}^{\infty} v(\log 1+v)^{2} \exp \left\{-v^{3 / 2}\right\} \sum_{u=1}^{\infty} \sum_{n}(u) \\
& \left.\ll \exp \{-L\} \Lambda(Z)=\Lambda(Z)^{2}\right)
\end{aligned}
$$

The three upper bounds (4.2), (4.4), and (4.10) now establish (1.8) and so complete the proofs of Theorem 3 and Theorem 1 .

\section{REFERENCES}

1. P.A. Catlin, Two problems in metric Diophantine approximation, I, J. Number Theory, 8 (1976), 282-288.

2. - Two problems in metric Diophantine approximation, II, J. Number Theory, 8 (1976), 289-297.

3. K. Chung, A Course in Probability Theory, Harcourt, Brace \& World, 1968.

4. R. J. Duffin and A. C. Schaeffer, Khintchine's problem in metric Diophantine approximation, Duke Math. J., 8 (1941), 243-255.

5. P. Erdös, On the distribution of convergents of almost all real numbers, J. Number Theory, 2 (1970), 425-441.

6. P. Gallagher, Approximation by reduced fractions, J. Math. Soc. of Japan, 13 (1961), 342-345.

7. A. Khintchine, Zur metrischen theorie der Diophantischen approximation, Math. Zeit., 24 (1926), 706-714.

Received April 29, 1977.

The University of Texas

AUstin, TX 78712 



\section{PACIFIC JOURNAL OF MATHEMATICS}

\section{EDITORS}

RICHARD ARENS (Managing Editor)

University of California

Los Angeles, CA 90024

Charles W. Curtis

University of Oregon

Eugene, OR 97403

C. C. MOORE

University of California

Berkeley, CA 94720

\section{J. DugundJI}

Department of Mathematics

University of Southern California

Los Angeles, CA 90007

R. FinN and J. Milgram

Stanford University

Stanford, CA 94305

\section{ASSOCIATE EDITORS}
E. F. BECKENBACH
B. H. NeumanN
F. WOLF
K. YosHIDA

\section{SUPPORTING INSTITUTIONS}

\author{
UNIVERSITY OF BRITISH COLUMBIA \\ CALIFORNIA INSTITUTE OF TECHNOLOGY \\ UNIVERSITY OF CALIFORNIA \\ MONTANA STATE UNIVERSITY \\ UNIVERSITY OF NEVADA, RENO \\ NEW MEXICO STATE UNIVERSITY \\ OREGON STATE UNIVERSITY \\ UNIVERSITY OF OREGON
}

\author{
UNIVERSITY OF SOUTHERN CALIFORNIA \\ STANFORD UNIVERSITY \\ UNIVERSITY OF HAWAII \\ UNIVERSITY OF TOKYO \\ UNIVERSITY OF UTAH \\ WASHINGTON STATE UNIVERSITY \\ UNIVERSITY OF WASHINGTON
}

The Supporting Institutions listed above contribute to the cost of publication of this Journal, but they are not owners or publishers and have no responsibility for its content or policies.

Mathematical papers intended for publication in the Pacific Journal of Mathematics should be in typed form or offset-reproduced, (not dittoed), double spaced with large margins. Please do not use built up fractions in the text of the manuscript. However, you may use them in the displayed equations. Underline Greek letters in red, German in green, and script in blue. The first paragraph or two must be capable of being used separately as a synopsis of the entire paper. Items of the bibliography should not be cited there unless absolutely necessary, in which case they must be identified by author and journal, rather than by item number. Manuscripts, in triplicate, may be sent to any one of the editors. Please classify according to the scheme of Math. Reviews, Index to Vol. 39. All other communications should be addressed to the managing editor, or Elaine Barth, University of California, Los Angeles, California, 90024.

50 reprints to each author are provided free for each article, only if page charges have been substantially paid. Additional copies may be obtained at cost in multiples of 50 .

The Pacific Journal of Mathematics is issued monthly as of January 1966. Regular subscription rate: $\$ 72.00$ a year (6 Vols., 12 issues). Special rate: $\$ 36.00$ a year to individual members of supporting institutions.

Subscriptions, orders for numbers issued in the last three calendar years, and changes of address should be sent to Pacific Journal of Mathematics, 103 Highland Boulevard, Berkeley, California, 94708. Older back numbers obtainable from Kraus Periodicals Co., Route 100, Millwood, NY 10546.

PUBLISHED BY PACIFIC JOURNAL OF MATHEMATICS, A NON-PROFIT CORPORATION

Printed at Kokusai Bunken Insatsusha (International Academic Printing Co., Ltd.). 8-8, 3-chome, Takadanobaba, Shinjuku-ku, Tokyo 160, Japan.

Copyright (C) 1978 by Pacific Journal of Mathematics Manufactured and first issued in Japan 


\section{Pacific Journal of Mathematics}

\section{Vol. 76, No. $2 \quad$ December, 1978}

Stephanie Brewster Brewer Taylor Alexander, Local and global convexity in complete Riemannian manifolds ...........................

Claudi Alsina i Català, On countable products and algebraic convexifications of probabilistic metric spaces ...............................

Joel David Berman and George Grätzer, Uniform representations of

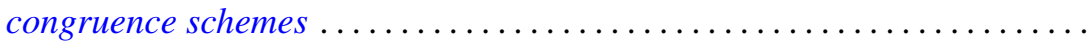

Ajit Kaur Chilana and Kenneth Allen Ross, Spectral synthesis in

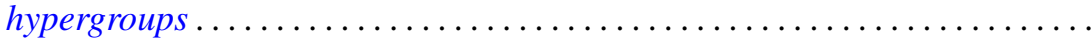

David Mordecai Cohen and Howard Leonard Resnikoff, Hermitian quadratic forms and Hermitian modular forms . .........................

Frank Rimi DeMeyer, Metabelian groups with an irreducible projective

representation of large degree .............................

Robert Ellis, The Furstenberg structure theorem .....................

Heinz W. Engl, Random fixed point theorems for multivalued mappings .......

William Andrew Ettling, On arc length sharpenings ..................

Kent Ralph Fuller and Joel K. Haack, Rings with quivers that are trees........

Kenneth R. Goodearl, Centers of regular self-injective rings ...............

John Gregory, Numerical algorithms for oscillation vectors of second order

differential equations including the Euler-Lagrange equation for

symmetric tridiagonal matrices.

Branko Grünbaum and Geoffrey Shephard, Isotoxal tilings

Myron Stanley Henry and Kenneth Leroy Wiggins, Applications of

approximation theory to differential equations with deviating

arguments

Mark Jungerman, The non-orientable genus of the n-cube .

Robert Richard Kallman, Only trivial Borel measures on $S_{\infty}$ are

quasi-invariant under automorphisms ................

Joyce Longman and Michael Rich, Scalar dependent algebras in the

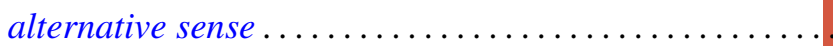

Richard A. Mollin, The Schur group of a field of characteristic zero ........ 471

David Pokrass, Some radical properties of rings with $(a, b, c)=(c, a, b) \ldots 479$

Margaret Shay and Paul Ruel Young, Characterizing the orders changed by

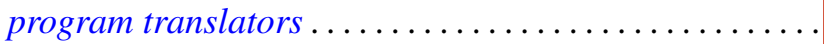

Jerrold Norman Siegel, On the structure of $B_{\infty}(F), F$ a stable space...

Surjeet Singh, (hnp)-rings over which every module admits a basic

submodule...

A. K. Snyder, Universal interpolating sets and the Nevanlinna-Pick property in

Banach spaces of functions...

Jeffrey D. Vaaler, On the metric theory of Diophantine approximation ... 